\title{
Cerebrovaskularne bolesti (Cerebrovascular Diseases)
}

Svetomir P. Ivanović (editor), Montenegrin Academy of Sciences and Arts, Podgorica, 2010, 598 pages; ISBN 978-86-7215-254-8; COBISS.CG-ID 16794896

Osman Sinanović, M.D., PhD

Professor of Neuropsychiatry

Medical School, University of Tuzla

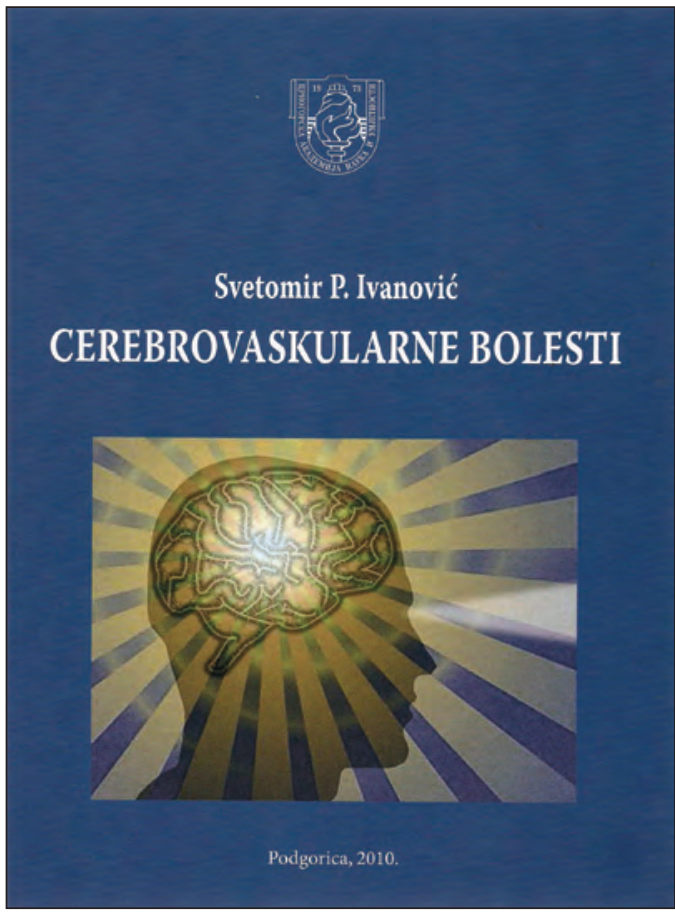

The book entitled Cerebrovascular Diseases, edited by Svetomir P. Ivanović, M.D., Ph.D., Academician of Montenegrin Academy of Sciences and Arts, Professor of Neurosurgery at the "US Medical School» in Belgrade (Serbia) is result of long lasting experience in the cerebrovascular field of this distinguished expert in the region. The book contains 32 chapters and has 36 contributors from different cerebrovascular subdisciplines from Serbia and Montenegro.
Stroke, whether of ischemic or hemorrhagic origin, is globally a major health burden. It is the second or third most common cause of death worldwide and most common cause of neurological disability in adults and second most important cause of dementia worldwide. According to WHO figures, global stroke deaths were 5.8 million in 2005 and are projected to increase to 6.5 million in 2015 and 7.8 million in 2030. Despite considerable advances, some aspects of the diagnostics and treatment of stroke remain controversial.

The main aim of the authors were to publish comprehensive basic and clinical knowledge including anatomy, physiology, pathophysiology, epidemiology, diagnostics, clinical presentations and different aspects of treatment and rehabilitation of all aspects of cerebrovascular diseases. The authors' aim has been accomplished. This textbook can serve as a very precise guide to all experts in the field of cerebrovascular disorders. Thanks to a very contemporary content, a very simple and in the same time very organized text, this book should be a basic and very helpful textbook for neurology, neurosurgery and physical medicine residents and specialists, but also for other specialists, residents and general practitioners. 